\title{
The Impact of Patient Interactive Systems on the Management of Pain in an Inpatient Hospital Setting: A Systematic Review
}

\author{
Raniah N. Aldekhyyel ${ }^{1,2}$ Caitlin J. Bakker ${ }^{3}$ Michael B. Pitt ${ }^{4}$ Genevieve B. Melton ${ }^{1,5,6,7}$
}

${ }^{1}$ Institute for Health Informatics, University of Minnesota, Minneapolis, Minnesota, United States

2 Medical Education Department, College of Medicine, King Saud University, Riyadh, Saudi Arabia

${ }^{3}$ Health Sciences Libraries, University of Minnesota, Minneapolis, Minnesota, United States

${ }^{4}$ Department of Pediatrics, University of Minnesota, Minneapolis, Minnesota, United States

${ }^{5}$ Department of Surgery, University of Minnesota, Minneapolis, Minnesota, United States

6 University of Minnesota Physicians, University of Minnesota,

Minneapolis, Minnesota, United States

${ }^{7}$ Fairview Health Services, Minneapolis, Minnesota, United States

Appl Clin Inform 2019;10:580-596.
Address for correspondence Genevieve B. Melton, MD, PhD, FACMI, Department of Surgery, University of Minnesota, MMC 450, 420 Delaware Street SE, Minneapolis, MN 55455, United States (e-mail: gmelton@umn.edu).

\section{Abstract}

Keywords

- pain management

- medical informatics applications

- patient participation

- outcome assessment
Background While some published literature exists on the use of interactive patient care systems, the effectiveness of these systems on the management of pain is unclear. To fill this gap in knowledge, we aimed to understand the impact and outcomes of pain management patient interactive systems in an inpatient setting.

Methods A systematic literature review was conducted across seven databases, and results were independently screened by two researchers. To extract relevant data, critical appraisal forms were developed and each paper was examined by two experts. Information included patient interactive system category, patient population and number of participants/samples, experiment type, and specific outcome measures.

Results Out of 58 full-text articles assessed for eligibility, 18 were eligible and included in the final qualitative synthesis. Overall, there were two main types of pain management interactive systems within the inpatient setting (standalone systems and integrated platform systems). While systems were diverse especially for integrated platforms, most reported systems were entertainment distraction systems. Reports examined a variety of outcome measures, including changes in patient-reported pain levels, patient engagement, user satisfaction, changes in clinical workflow, and changes in documentation. In the 13 systems measuring pain scores, 12 demonstrated a positive impact on pain level scores.

Conclusion Pain management systems appear to be effective in lowering patient level scores, but research comparing the effectiveness and efficacy of one type of interactive system versus another in the management of pain is needed. While not conclusive, pain management systems integrated with other technology platforms show potentially promising effects with improving patient communication, education, and self-reporting. received

April 7, 2019

accepted after revision

June 19, 2019 (c) 2019 Georg Thieme Verlag KG

Stuttgart · New York
DOI https://doi.org/ 10.1055/s-0039-1694002. ISSN 1869-0327. 


\section{Background and Significance}

The management of acute pain in a hospital setting is a multifaceted, complex process made no less complicated by the fact that each patient's experiences with pain is subjective and unique. Providers are being taught to consider pain as the "sixth vital sign," with this emphasis reinforced by the priority placed on patient feedback on how their pain was managed on the Hospital Consumer Assessment of Healthcare Providers and Systems survey, the most widely used metric for patient satisfaction. ${ }^{1}$ Successful pain management goes beyond simply reacting to acute pain with medication, as evidenced by the Joint Commission, hospitals are required to involve patients in pain management treatment planning, to educating patients on pain management discharge plans, and to providing nonpharmacologic pain treatment modalities. ${ }^{2}$

Individuals experience pain differently, with demographic factors such as race/ethnicity, age, and gender ${ }^{3,4}$ and psychological factors such as anxiety and past experiences ${ }^{5,6}$ possibly impacting how patients perceive and respond to the treatment of pain. Complicating things further, patient satisfaction with pain control is not the same as patients simply endorsing the absence of pain. In fact, Pronovost and colleagues showed that patients were more likely to rate their overall satisfaction high if they perceived that their care providers did everything possible to control pain, regardless of how much pain the patients were actually in. ${ }^{7}$

In response to this need for individualized approaches, hospitals are turning to information technology to develop and implement systems to support an optimal multifaceted pain management process. Many of these applications have focused on automation of the pain assessment and documentation process, ${ }^{8-10}$ or on the use of mobile applications to engage patients in tracking and managing their pain. ${ }^{11,12}$ Web-based intervention systems create opportunities to enhance patient education and engagement via interactivity, ${ }^{13,14}$ defined as the "extent to which users can participate in modifying the form and content of a mediated environment in real time." 15 For the purposes of this review, we refer to inpatient interactive systems in a broad sense of patient/ caregiver usage of technology that delivers one or more of the following features: health self-management, communication, education, or entertainment/distraction services in an inpatient hospital setting. These systems are designed to enhance or promote patient engagement through interacting with the system's components through specific electronic devices, inpatient portals, or inpatient television screens.

Patients who are more actively involved in their health care experience better health outcomes, lower health costs, and higher levels of satisfaction. ${ }^{16-18}$ The use of patient interactive systems in the management of pain have been reported in the literature since the early 2000s when virtual reality (VR) systems were introduced to distract patients during painful therapy or treatment. In more recent years, the use of these systems has extended beyond a means of distraction to serve as a platform for patient-provider communication and enhance access to patient-standardized patient education. These systems can potentially allow for a more patient-centered care approach and an improved clinical workflow.

Previous systematic reviews explored patient engagement systems, ${ }^{19}$ demonstrated the effects of patient interactive systems on patient engagement, ${ }^{20}$ and their impact on patients' self-management of health, such as diabetes, ${ }^{21}$ asthma, ${ }^{22}$ weight loss, ${ }^{23}$ and smoking cessation. ${ }^{24}$ To our knowledge, no review has been conducted on the impact of patient interactive systems on pain management, particularly in an inpatient setting.

\section{Objectives}

We aimed to summarize the current state of scientific literature regarding the use of patient interactive systems designed for the management of pain within an inpatient hospital setting. Specifically, we sought to determine whether patient engagement through the use of interactive systems for pain management leads to improvements in clinical care, clinical workflows, patient outcomes, or user satisfaction.

\section{Methods}

\section{Search Strategy}

We systematically searched the literature to capture all publications relating to the impact of patient engagement and the use of interactive systems on clinical care, workflows, patientreported outcomes, and user satisfaction. We registered the review protocol in PROSPERO, ${ }^{25}$ and conducted searches across 8 databases: MEDLINE and Embase (both via Ovid), Cochrane Library (via Wiley), Web of Science, Scopus, Global Index Medicus, ClinicalTrials.gov, and WHO ICTRP. Additionally, we consulted the reference lists of relevant systematic reviews and hand-searched conference proceedings. In accordance with the Methodological Expectations for Cochrane Intervention Reviews guidelines, ${ }^{26}$ we employed a combination of controlled vocabulary and natural language and we placed no limitations on date of publication or language. A complete search strategy is available in the - Supplementary Appendix (available in the online version). Searches were first conducted in June 2018 and results were updated in May 2019. Results were compiled and de-duplicated using EndNote (Version X7). ${ }^{27}$

Studies that describe the implementation or use of a patient interactive system for the management of pain in an inpatient hospital setting were included in this review. Exclusion criteria included reports exclusively in outpatient or home care settings, not utilizing an interactive pain management system, and not engaged in pain management activities. Articles that did not report original data, such as narrative reviews or opinion pieces, were also excluded.

\section{Screening and Study Selection}

Three experienced researchers (health information professional, health informatics professional, and medical doctor) conducted the screening. Two independent screeners (R.A. and M.P.) reviewed each title and abstract for inclusion using Rayyan, a Web application that supports collaboration among researchers during screening and study selection. ${ }^{28}$ 
Where discrepancies existed between the two screeners, a decision was reached through discussion or, where necessary, by a third screener (C.B.). Full-text screening followed title and abstract screening, again with the two independent screeners determining inclusion. Screeners recorded rationale for exclusion, which is reported in a Preferred Reporting Items for Systematic Reviews and Meta-Analyses diagram (-Fig. 1)..$^{29}$

\section{Data Extraction and Classification}

We developed data extraction forms that captured whether articles reported on specific outcome measures: (1) changes in clinical workflow; measuring a set of outcomes measures, which may include pre- and postcomparison studies; (2) patient engagement; measuring dimensions of patient participation, patient activation, patient engagement, patient moti- vation, or self-efficacy through patient reports, validated surveys such as the Patient Activation Measure, the Altarum Consumer Engagement Measure, or measures defined by authors such as "sense of presence" ${ }^{30}$; (3) user satisfaction (patient, parent, or nurse); measured through patient reports, validated surveys, or qualitative measures such as patient interviews; (4) changes in patient-reported pain level scores; measured through a validated clinical pain assessment tool; and (5) changes in electronic health record (EHR) pain documentation; measuring a set of outcomes measures, which include pre- and postcomparison studies.

Where available, we collected further information on the mechanism for gathering that data, such as the use of a validated survey or measurement tool. We also recorded the type of interactive tool being utilized, the number of participants, and whether it was an adult or pediatric population.
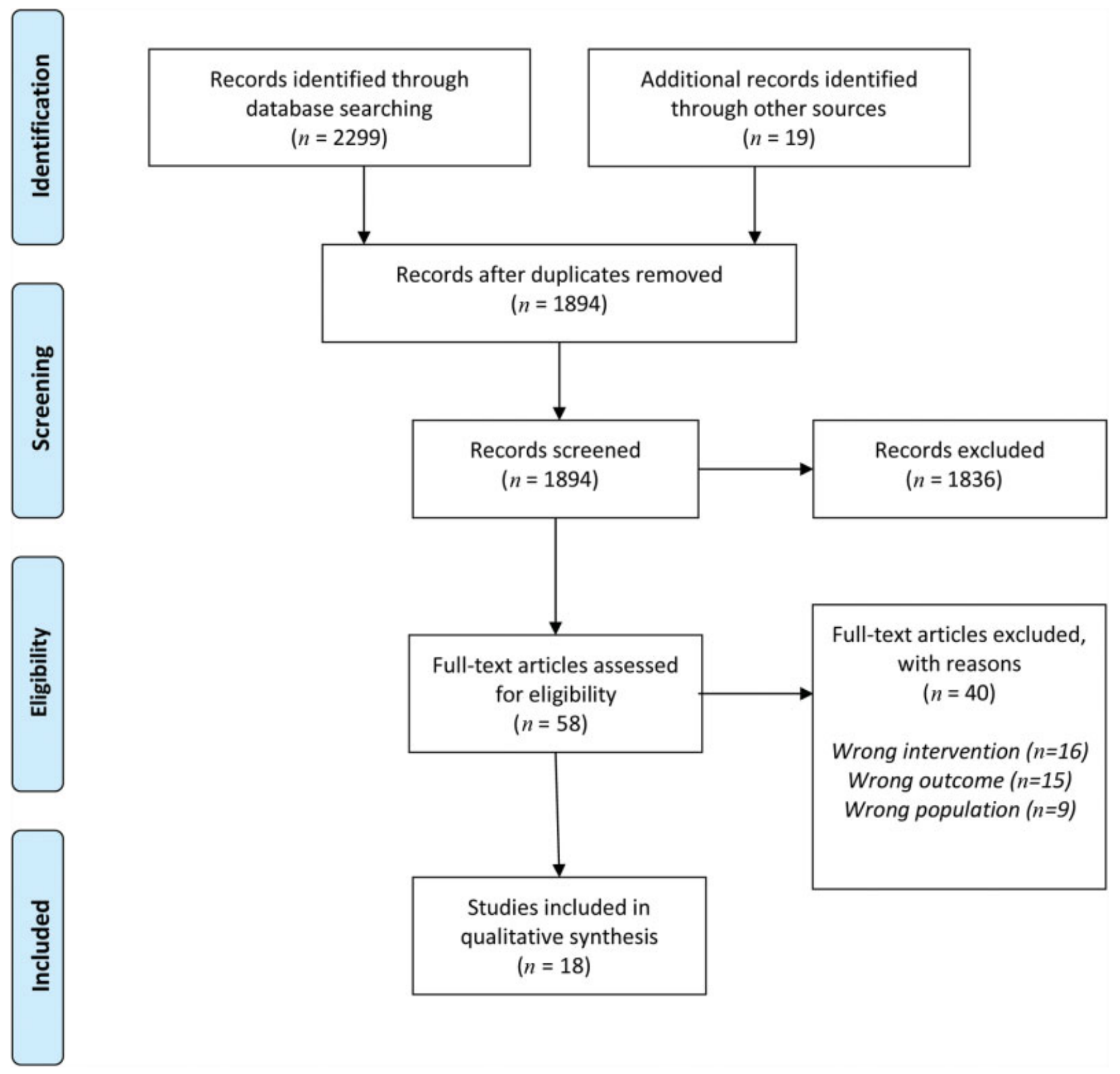

Studies included in

qualitative synthesis

$(n=18)$

Fig. 1 Preferred Reporting Items for Systematic Reviews and Meta-Analyses (PRISMA) diagram. 
One author developed the forms, which were then piloted and refined by the other two authors.

Risk of bias was assessed using ROBINS-I (Risk Of Bias In NonRandomized Studies - of Interventions) for nonrandomized studies and GRADE (Grading of Recommendations Assessment, Development, and Evaluation) for randomized studies. ${ }^{31,32}$ Two independent screeners performed data extraction and risk of bias assessment using Qualtrics. ${ }^{33}$ Discrepancies were resolved through discussion with a third screener. Due to the heterogeneity of the studies, meta-analysis was not possible.

\section{Results}

The combined search strategies identified 1,894 electronic records for the title and abstract screening phase, which yielded 58 potentially eligible studies for full-text screening. Of these, 18 met the study inclusion criteria and were included in the final qualitative synthesis phase (-Fig. 1).

Ten studies explored the use of interactive pain management systems in an adult population, four in a pediatric population, and three included both an adult and pediatric population. There were 13 randomized controlled trials (RCTs) and 5 non-RCTs. All studies were published in English and three studies were conducted outside of the United States.

\section{Study Quality}

Results of the risk of bias assessment for RCTs are reported in - Fig. 2. Only one trial was at low risk of bias for all quality criteria $^{34}$ while all others included some unclear or high risk of bias for some quality criteria. The risk of bias assessment for nonrandomized studies is reported in -Fig. 3. Overall, the majority of studies were found to have an unclear risk of bias.

\section{Types of Pain Management Interactive Systems}

There were two main types of pain interactive systems described: standalone systems (systems designed to include one specific function) and platform systems (systems designed to include more than one function). These are summarized in - Table 1 and described further below.

\section{Standalone Interactive Systems}

There were 14 stand-alone interactive systems described, of which 9 focused on the use of VR designed to distract patients from abdominal pain, ${ }^{35}$ or burn patients from pain during physical therapy sessions ${ }^{30,34,36-38}$ or wound/dressing changes. ${ }^{39-41}$ One system described using interactive music therapy, ${ }^{42}$ two other described a Web-based virtual nurse designed for patient-provider communication and patient education, ${ }^{42,43}$ and one described a bedside educational multimedia program intervention accessed through iPads. ${ }^{44} \mathrm{Fi}-$ nally, one paper described a personalized patient-controlled analgesia (PCA) device for oral medications. ${ }^{45}$

\section{VR Distraction Systems}

The use of VR systems in the management of pain offers a psychologically based approach to help minimize pain. These systems are computer-generated environments, where “... patients typically use a head-mounted three-dimensional visual screen to interact with a computer environment to draw attention away from their pain." ${ }^{39}$ Carrougher et al described why VR is helpful in reducing patient pain by explaining that "...individuals have a limited amount of attention that can be divided between incoming stimuli, and pain, which requires attention. Because VR is a highly attention-grabbing experience, it can be an effective psychological pain control technique." 36

Hoffman et al published the earliest studies (conducted in 2000 and 2001) that examined the use of VR by burn patients during physical therapy sessions. ${ }^{30,38}$ Studies that followed examined the efficacy of using VR in controlling pain in children with acute burn injuries, ${ }^{41}$ factors that influenced the efficacy of VR in distracting patients during physical therapy, ${ }^{36,37}$ and examined whether preprocedural VR-guided relaxation added to morphine-reduced pain severity during dressings changes in burn patients. ${ }^{40}$ Later studies focused on testing the feasibility and potential effects of a low-cost VR systems in reducing patient pain during physiotherapy in a developing country, ${ }^{34}$ examined the repeated use of VR to control pain during wound dressing changes, ${ }^{39}$ and studied the impact of VR on patients suffering from acute abdominal pain. ${ }^{35}$

\section{Interactive Music}

Among the reviewed studies that examined the use of entertainment/distraction in the management of pain during therapy, Li et al was the only study that described the use of an interactive music therapy intervention. ${ }^{42}$ The intervention was designed to allow patients to choose their preferred music, control music volume, and listen through a personal headphone connected to an MP3 player. The authors reported the effects of the intervention on reducing pain after radical mastectomy in cancer patients in China.

\section{Communication/Educational Systems}

There were three studies that examined and evaluated the development of an educational/communication system, which is designed to deliver personalized pain-relieving educational videos through different types of output devices. Two studies investigated the development of a virtual nursing Web-based intervention to improve pain relief in cardiac surgery patients and reported on the preliminary effects of the system. ${ }^{42,44}$ The Web application generates reflective personalized activities and specific educational messages designed for patients based on their beliefs and attitudes. The system messages are transmitted through videos of a virtual nurse, animations, stories, and texts. The third study evaluated the efficacy of a bedside multimedia intervention accessed through iPads. The intervention is designed to engage patients in their recovery process after total knee replacement surgery. ${ }^{44}$

\section{Personalized PCA Device}

One study described a novel oral PCA dispenser providing ondemand pain medication at the bedside. The device has three main features: a drug dispensing unit, a radiofrequency identification wristband used for patient's registration, and a PillBox; "a patient-specific, mouth-actuated, disposable receptacle from which the patient receives the pill." 45 
A

B
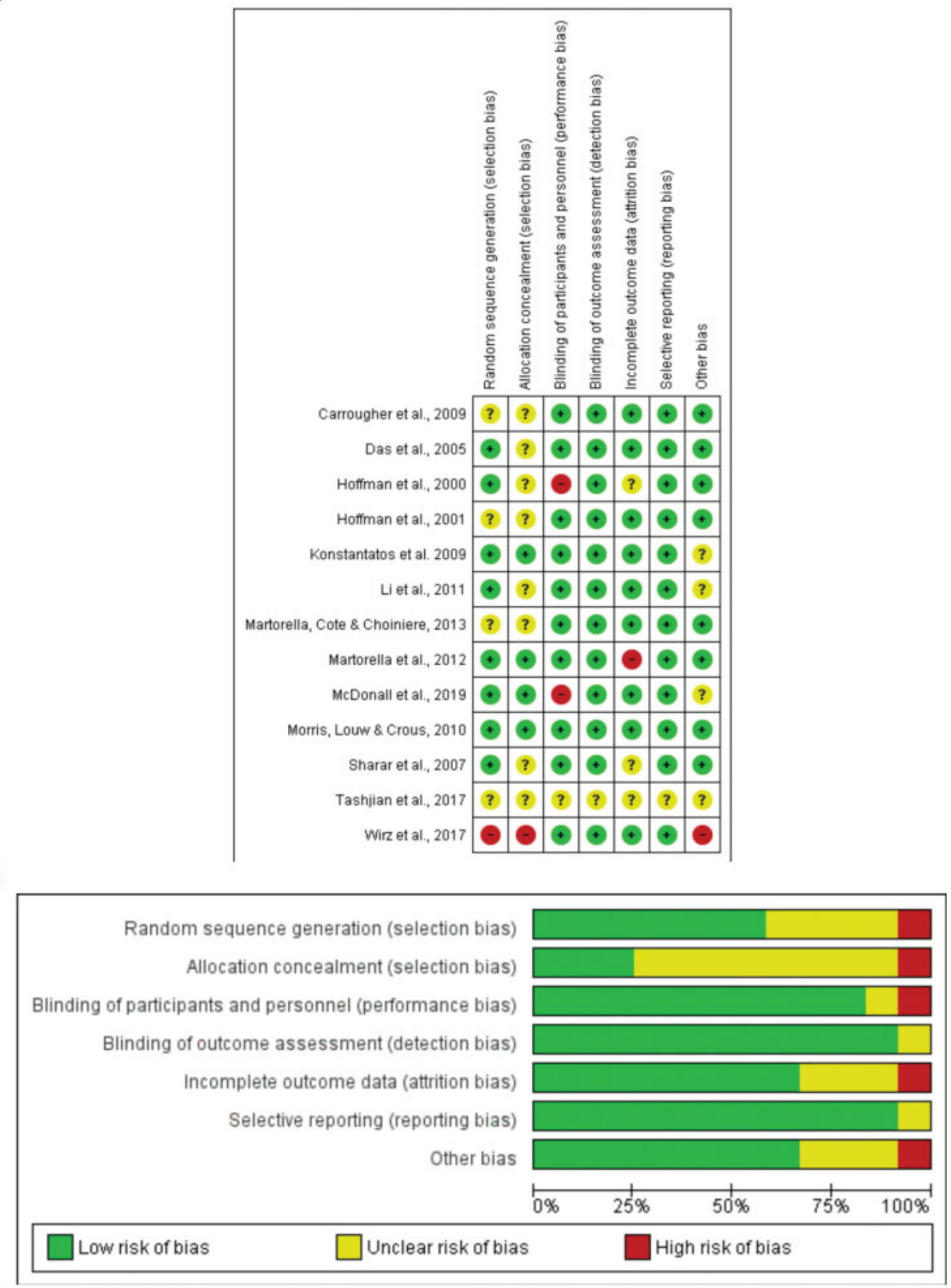

Fig. 2 The risk of bias assessment with Grading of Recommendations Assessment, Development, and Evaluation (GRADE) for randomized controlled trials. (A) Risk of bias summary. (B) Risk of bias graph.

\section{Interactive Platform Systems}

Interactive platform systems are systems which include interactive prompts meant to better engage patients in their care during a hospital encounter and improve their patient experience. These systems display standardized patient education material, care management, patientprovider communication, and/or entertainment features, which are accessed through patient-facing interfaces 
A

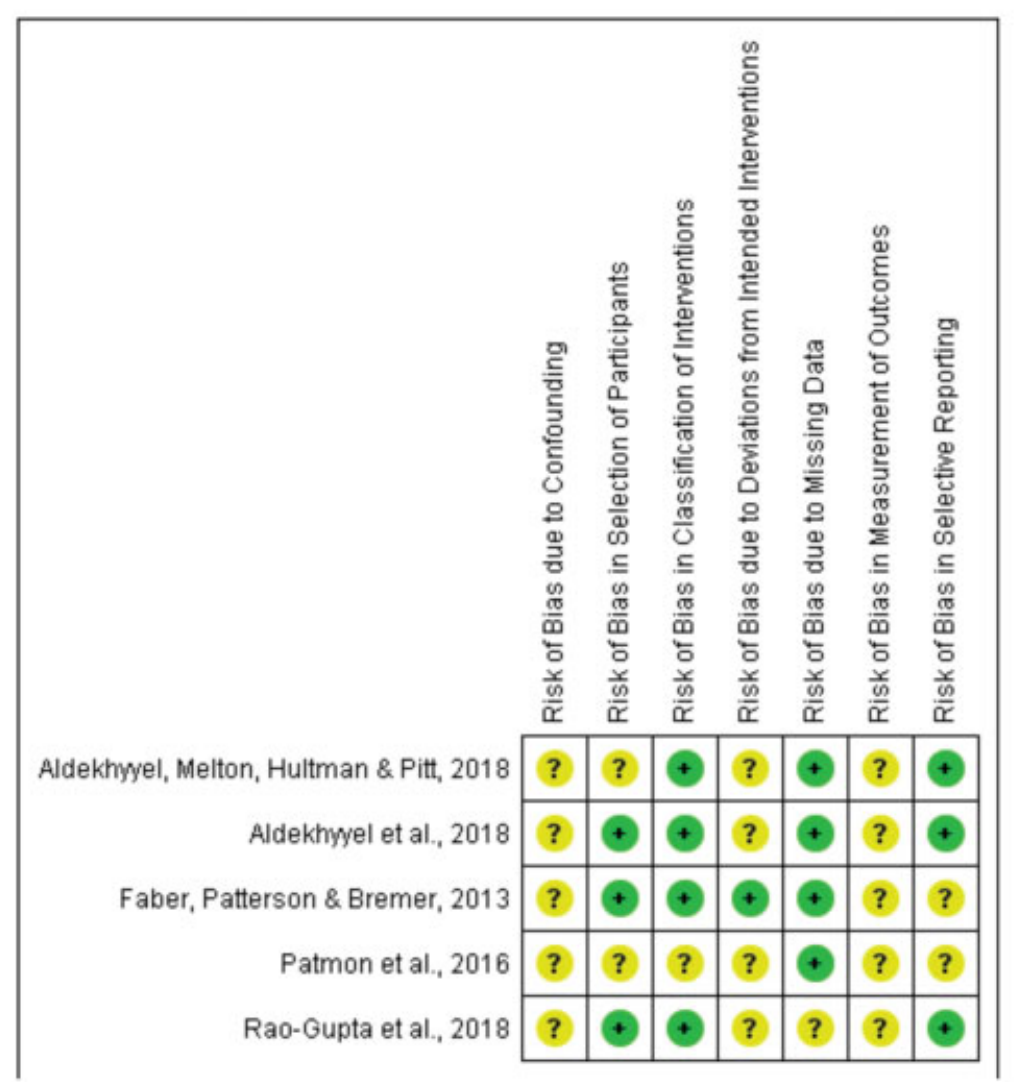

B

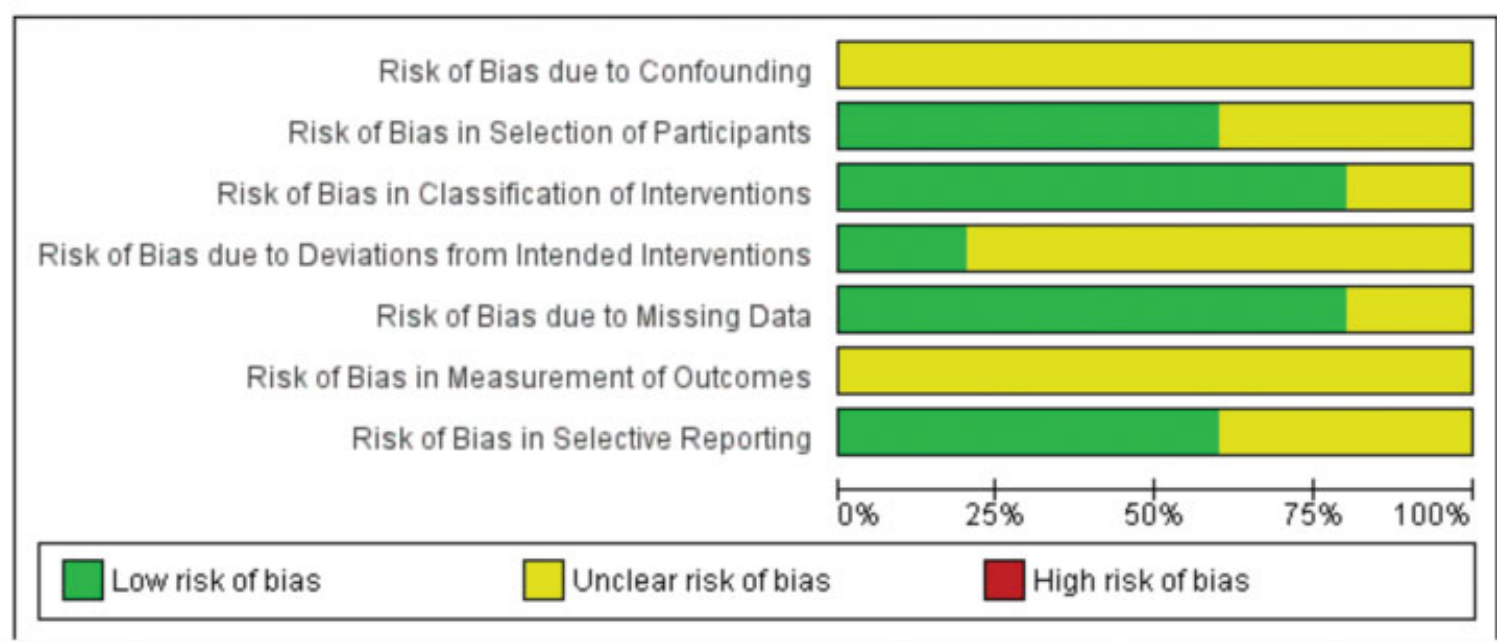

Fig. 3 The risk of bias assessment with the Risk Of Bias In Non-Randomized Studies - of Interventions (ROBINS-I) tool for nonrandomized controlled trials. (A) Risk of bias summary. (B) Risk of bias graph.

such as inpatient bedside television screens or portable devices. Other systems' capabilities may include EHR integration and real-time patient-provider communication and alerts. ${ }^{46}$

There were four studies describing the use of interactive platform systems built to support patient engagement and timely nursing pain reassessment documentation practices. Two described the effects of integrating an interactive platform system, accessed through the inpatient television screens, with the hospital's EHR system, medication dispensing machine, and the nursing staff call system. ${ }^{47,48}$ The third study described a similar interactive platform system for bedside pain reporting through the television; however, the system was not integrated with the nursing staff call system. ${ }^{49}$ The fourth study described the perceptions of nurses who use an interactive system via iPads and 
Table 1 Details of reviewed studies

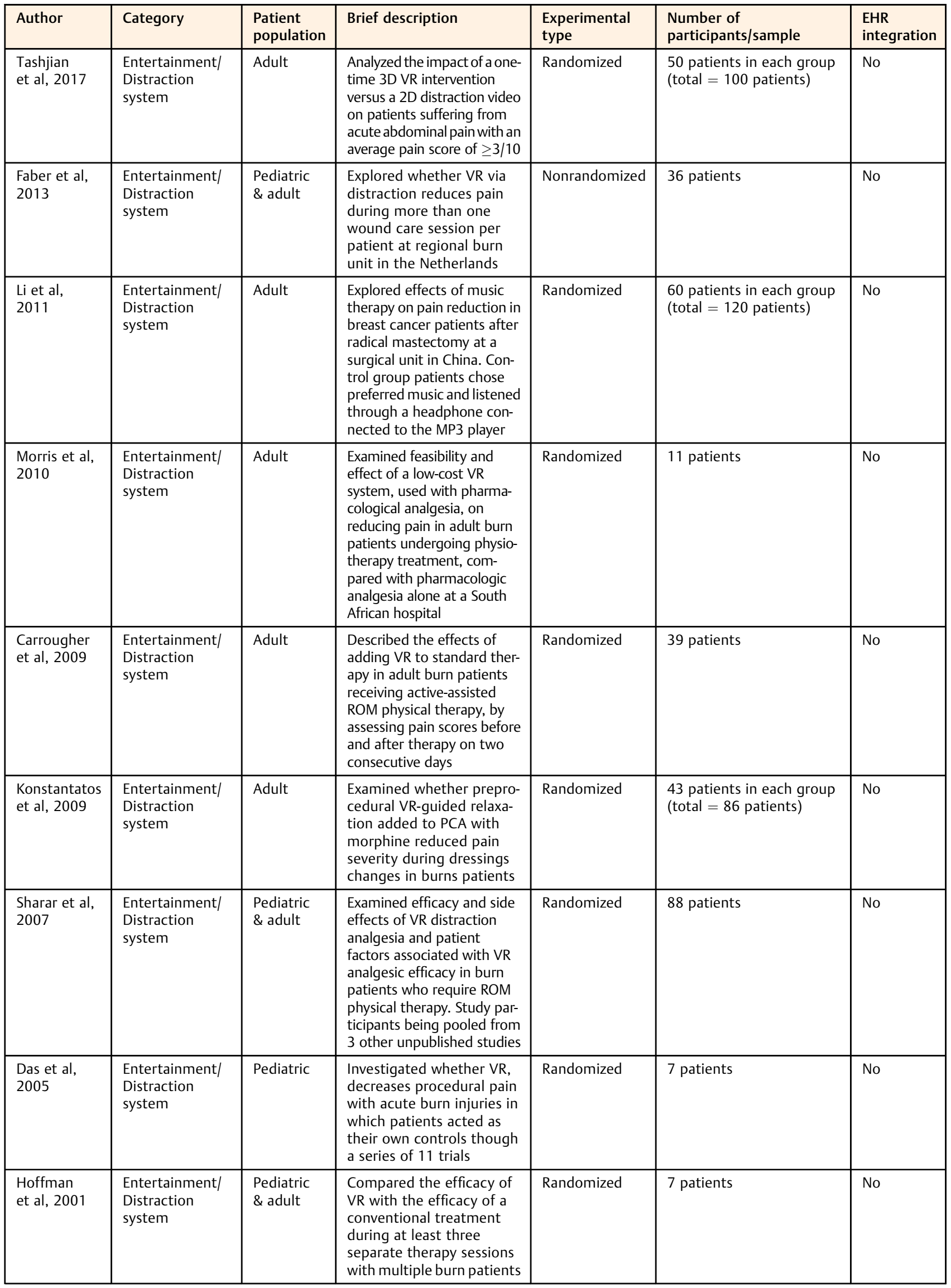


Table 1 (Continued)

\begin{tabular}{|c|c|c|c|c|c|c|}
\hline Author & Category & $\begin{array}{l}\text { Patient } \\
\text { population }\end{array}$ & Brief description & $\begin{array}{l}\text { Experimental } \\
\text { type }\end{array}$ & $\begin{array}{l}\text { Number of } \\
\text { participants/sample }\end{array}$ & $\begin{array}{l}\text { EHR } \\
\text { integration }\end{array}$ \\
\hline $\begin{array}{l}\text { Hoffman } \\
\text { et al, } 2000\end{array}$ & $\begin{array}{l}\text { Entertainment/ } \\
\text { Distraction } \\
\text { system }\end{array}$ & Adult & $\begin{array}{l}\text { Explored use of VR to } \\
\text { distract burn patients from } \\
\text { pain during physical therapy } \\
\text { treatment. Each patient } \\
\text { spent } 3 \text { min of physical } \\
\text { therapy with no VR and } \\
3 \text { min of physical therapy } \\
\text { with VR }\end{array}$ & Randomized & 12 patients & No \\
\hline $\begin{array}{l}\text { Martorella } \\
\text { et al, } 2012\end{array}$ & $\begin{array}{l}\text { Communication/ } \\
\text { Educational } \\
\text { system }\end{array}$ & Adult & $\begin{array}{l}\text { Investigated the preliminary } \\
\text { effects of a virtual nursing } \\
\text { Web-based intervention to } \\
\text { improve pain relief in } \\
\text { patients undergoing cardiac } \\
\text { surgery. The intervention } \\
\text { includes a preoperative } \\
30 \text {-min Web-based session } \\
\text { and } 2 \text { brief face-to-face post- } \\
\text { operative sessions. It gener- } \\
\text { ates reflective activities and } \\
\text { specific personalized educa- } \\
\text { tional messages transmitted } \\
\text { through videos of a virtual } \\
\text { nurse, animations, stories, } \\
\text { and texts }\end{array}$ & Randomized & $\begin{array}{l}30 \text { patients in each group } \\
\text { (total }=60 \text { patients) }\end{array}$ & No \\
\hline $\begin{array}{l}\text { Martorella } \\
\text { et al, } 2013\end{array}$ & $\begin{array}{l}\text { Communication/ } \\
\text { Educational } \\
\text { system }\end{array}$ & Adult & $\begin{array}{l}\text { Reported the development, } \\
\text { validation, feasibility and } \\
\text { acceptability of a virtual } \\
\text { nursing Web-based interven- } \\
\text { tion for postoperative pain } \\
\text { self-management in adults } \\
\text { scheduled for cardiac } \\
\text { surgery. The intervention } \\
\text { includes a preoperative } \\
\text { 30-min Web-based session } \\
\text { and } 2 \text { brief face-to-face post- } \\
\text { operative sessions. It gener- } \\
\text { ates reflective activities and } \\
\text { specific personalized educa- } \\
\text { tional messages transmitted } \\
\text { through videos of a virtual } \\
\text { nurse, animations, stories, } \\
\text { and texts }\end{array}$ & Randomized & $\begin{array}{l}30 \text { patients in each group } \\
\text { (total }=60 \text { patients) }\end{array}$ & No \\
\hline $\begin{array}{l}\text { McDonall } \\
\text { et al, } 2019\end{array}$ & $\begin{array}{l}\text { Communication/ } \\
\text { Educational } \\
\text { system }\end{array}$ & Adult & $\begin{array}{l}\text { Evaluated the efficacy of a } \\
\text { bedside multimedia inter- } \\
\text { vention accessed through } \\
\text { iPads, designed to engage } \\
\text { patients in their recovery } \\
\text { process after total knee } \\
\text { replacement surgery. The } \\
\text { intervention contains } \\
\text { tailored educational infor- } \\
\text { mation based on each day of } \\
\text { recovery and specific patient } \\
\text { recovery goals, which are } \\
\text { facilitated by clinicians }\end{array}$ & Randomized & $\begin{array}{l}104 \text { patients in the } \\
\text { intervention group } \\
\text { (total }=241 \text { ) }\end{array}$ & No \\
\hline $\begin{array}{l}\text { Wirz et al, } \\
2017\end{array}$ & $\begin{array}{l}\text { Personalized } \\
\text { PCA device }\end{array}$ & Adult & $\begin{array}{l}\text { Evaluated the safety, } \\
\text { efficacy, and usability of a } \\
\text { novel bedside PCA } \\
\text { dispenser. The device has } \\
\text { three main features: a drug } \\
\text { dispensing unit, a radio- } \\
\text { frequency identification } \\
\text { wristband used for } \\
\text { patient's registration, and } \\
\text { a PillBox, which is a patient- } \\
\text { specific, mouth-actuated, } \\
\text { disposable receptacle, } \\
\text { from which the patient } \\
\text { receives the pill }\end{array}$ & Randomized & $\begin{array}{l}27 \text { patients in } \\
\text { the test group } \\
\text { (total }=70 \text { patients) }\end{array}$ & No \\
\hline
\end{tabular}

(Continued) 
Table 1 (Continued)

\begin{tabular}{|c|c|c|c|c|c|c|}
\hline Author & Category & $\begin{array}{l}\text { Patient } \\
\text { population }\end{array}$ & Brief description & $\begin{array}{l}\text { Experimental } \\
\text { type }\end{array}$ & $\begin{array}{l}\text { Number of } \\
\text { participants/sample }\end{array}$ & $\begin{array}{l}\text { EHR } \\
\text { integration }\end{array}$ \\
\hline $\begin{array}{l}\text { Aldekhyyel } \\
\text { et al, } 2018\end{array}$ & $\begin{array}{l}\text { Interactive } \\
\text { platform } \\
\text { system }\end{array}$ & Pediatric & $\begin{array}{l}\text { Described the implementa- } \\
\text { tion of a pain management } \\
\text { interactive tool, which allows } \\
\text { for real-time patient- } \\
\text { reported pain assessments } \\
\text { through inpatient television } \\
\text { screens, integrated with the } \\
\text { hospital's EHR, medication } \\
\text { dispensing machine and the } \\
\text { nursing phones. The study } \\
\text { measured the effects of } \\
\text { implementation by extract- } \\
\text { ing pre- and postimplemen- } \\
\text { tation pain assessment data } \\
\text { ( } 22 \text { mo) from the EHR }\end{array}$ & Nonrandomized & $\begin{array}{l}56,931 \text { patient records } \\
\text { ( } 2,447 \text { unique) }\end{array}$ & Yes \\
\hline $\begin{array}{l}\text { Aldekhyyel } \\
\text { et al, } 2018\end{array}$ & $\begin{array}{l}\text { Interactive } \\
\text { platform } \\
\text { system }\end{array}$ & Pediatric & $\begin{array}{l}\text { Described end-users' (nurses } \\
\text { and parents) perspectives on } \\
\text { using a pain management } \\
\text { interactive tool to report pain } \\
\text { using a mixed-methods } \\
\text { approach. The tool } \\
\text { integrated } 4 \text { standalone } \\
\text { technologies (EHR, } \\
\text { medication dispensing } \\
\text { machine, nurse phones, and } \\
\text { inpatient TV screens) }\end{array}$ & Nonrandomized & $\begin{array}{l}30 \text { parents and } 59 \text { nurses } \\
\text { (total }=89 \text { users) }\end{array}$ & Yes \\
\hline $\begin{array}{l}\text { Patmon } \\
\text { et al, } 2016\end{array}$ & $\begin{array}{l}\text { Interactive } \\
\text { platform } \\
\text { system }\end{array}$ & Adult & $\begin{array}{l}\text { Described the perceptions of } \\
\text { nurses who use an interactive } \\
\text { tool in their daily work. The } \\
\text { tool is built for patient-pro- } \\
\text { vider communication, } \\
\text { patient access to the EHR, } \\
\text { patienteducation, and } \\
\text { distraction therapy. It is } \\
\text { accessed through iPads in } \\
\text { the outpatient clinics and } \\
\text { television screens in the } \\
\text { inpatient rooms }\end{array}$ & Nonrandomized & 38 nurses & No \\
\hline $\begin{array}{l}\text { Rao-Gupta } \\
\text { et al, } 2018\end{array}$ & $\begin{array}{l}\text { Interactive } \\
\text { platform } \\
\text { system }\end{array}$ & Pediatric & $\begin{array}{l}\text { Described a quality improve- } \\
\text { ment project to develop new } \\
\text { workflows to integrate an } \\
\text { interactive patient care } \\
\text { technology system } \\
\text { (designed for the manage- } \\
\text { ment of pain accessed } \\
\text { through the inpatient } \\
\text { television screens) with the } \\
\text { hospital's automated } \\
\text { medication dispensing } \\
\text { system and integrate the } \\
\text { system with the hospital's } \\
\text { EHR }\end{array}$ & Nonrandomized & NA & Yes \\
\hline
\end{tabular}

Abbreviations: 2D, two-dimensional; 3D, three-dimensional; EHR, electronic health record; PCA, patient-controlled analgesia; ROM, range of motion; $\mathrm{VR}$, virtual reality.

televisions in their daily work. ${ }^{50}$ This system was mainly built to support patient-provider communication and patient access to the EHR.

\section{Outcome Measures}

We extracted 28 outcome measures from the 18 papers ranging between one and four outcomes per study. These outcomes mapped to five themes: (1) changes in patientreported pain levels; (2) patient engagement; (3) user satisfaction; (4) changes in clinical workflow; and (5) changes in clinical documentation practices.

\section{Standalone Interactive Systems}

\section{VR and Interactive Music Distraction Systems}

Studies that described the use of VR and interactive music as an entertainment/distraction intervention for the management of pain all reported changes in patient-reported pain levels, as a main outcome measure, using a Visual, Graphical, Numeric, or a modified Faces Pain Rating Scale. A total of 8 out of 9 VR distraction pain management intervention studies reported a statistically significant decrease in patient-reported pain level scores (- Table 2). ${ }^{30,34-39,41}$ Konstantatos et al was the only 
Table 2 Reported outcome measures from reviewed studies

\begin{tabular}{|c|c|c|c|c|c|}
\hline \multirow[t]{2}{*}{ Author } & \multirow[t]{2}{*}{ Category } & \multicolumn{4}{|c|}{ Outcome measures } \\
\hline & & \multicolumn{2}{|c|}{ Main outcome } & \multirow{2}{*}{$\begin{array}{l}\text { Measurement } \\
\text { Pre- and postintervention pain } \\
\text { scores }\end{array}$} & \multirow[b]{2}{*}{$\begin{array}{l}\text { Main findings } \\
\text { Mean pain reduction in VR cohort was } \\
\text { greater than in controls ( }-1.3 \text { vs. }-0.6 \\
\text { points; } p=0.008 \text { ). In Gl subgroups, } \\
\text { mean pain reduction in VR cohort } \\
\text { (pre }=5.72, \text { post }=4.18 ; \\
p=0.016) \text {. Multivariable regression } \\
\text { analysis adjusting for age, race, eth- } \\
\text { nicity, and gender, VR was a predictor } \\
\text { of pain reduction } \\
\text { independent of pain origin } \\
(\beta \text { coefficient }=-0.65 \text { points; } \\
p=0.05)\end{array}$} \\
\hline $\begin{array}{l}\text { Tashjian } \\
\text { et al, } 2017\end{array}$ & $\begin{array}{l}\text { Entertainment/ } \\
\text { Distraction } \\
\text { system }\end{array}$ & $1-$ & $\begin{array}{l}\text { Changes in patient- } \\
\text { reported pain levels }\end{array}$ & & \\
\hline $\begin{array}{l}\text { Faber et al, } \\
2013\end{array}$ & $\begin{array}{l}\text { Entertainment/ } \\
\text { Distraction } \\
\text { system }\end{array}$ & $1-$ & $\begin{array}{l}\text { Changes in patient- } \\
\text { reported pain levels }\end{array}$ & $\begin{array}{l}\text { Visual Analog Thermometer } \\
\text { ( } 10 \mathrm{~cm} \text { tall burn-specific pain } \\
\text { rating device) }\end{array}$ & $\begin{array}{l}\text { Pain ratings during wound } \\
\text { debridement were statistically } \\
\text { lower when patients were in VR on } \\
\text { days } 1,2 \text {, and } 3 \text {, and although not } \\
\text { significant beyond day } 3 \text {, the } \\
\text { pattern of results from days } 4,5 \text {, } \\
\text { and } 6 \text { were consistent with the } \\
\text { notion that VR continues to reduce } \\
\text { pain when used repeatedly }\end{array}$ \\
\hline $\begin{array}{l}\text { Li et al, } \\
2011\end{array}$ & $\begin{array}{l}\text { Entertainment/ } \\
\text { Distraction } \\
\text { system }\end{array}$ & $1-$ & $\begin{array}{l}\text { Changes in patient- } \\
\text { reported pain levels }\end{array}$ & $\begin{array}{l}\text { General Questionnaire and } \\
\text { Chinese version of Short-Form of } \\
\text { McGill Pain Questionnaire. Visual } \\
\text { Analogue Scale, and Present Pain } \\
\text { Intensity scores }\end{array}$ & $\begin{array}{l}\text { Pain scores measured at baseline } \\
\text { and } 3 \text { posttests. Music therapy } \\
\text { reduced Pain Rating Index } \\
\text { (PRI-total) score in intervention } \\
\text { group compared with control } \\
\text { group with a mean difference } \\
\text { of }-2.38,-2.41 \text {, and }-1.87 \text { for the } \\
1 \text { st, } 2 \text { nd, and } 3 \text { rd posttests, } \\
\text { respectively }\end{array}$ \\
\hline $\begin{array}{l}\text { Morris et al, } \\
2010\end{array}$ & $\begin{array}{l}\text { Entertainment/ } \\
\text { Distraction } \\
\text { system }\end{array}$ & $1-$ & $\begin{array}{l}\text { Changes in patient- } \\
\text { reported pain levels }\end{array}$ & Numeric Pain Rating Scale & $\begin{array}{l}\text { Patients reported a marginal } \\
(p=0.06) \text { to }(p=0.13) \text { differ- } \\
\text { ence between the two sessions } \\
\text { (analgesia with VR and analgesia } \\
\text { without VR) in reducing pain }\end{array}$ \\
\hline \multirow[t]{2}{*}{$\begin{array}{l}\text { Carrougher } \\
\text { et al, } 2009\end{array}$} & \multirow[t]{2}{*}{$\begin{array}{l}\text { Entertainment/ } \\
\text { Distraction } \\
\text { system }\end{array}$} & $1-$ & Patient engagement & 0-100 mm Graphic Rating Scale & $\begin{array}{l}\text { VR object "presence" was mea- } \\
\text { sured. Approximately half of the } \\
\text { patients ( } 51.3 \% \text { ) rated their level of } \\
\text { presence at greater than } 35 \mathrm{~mm}\end{array}$ \\
\hline & & $2-$ & $\begin{array}{l}\text { Changes in patient- } \\
\text { reported pain levels }\end{array}$ & 0-100 mm Graphic Rating Scale & $\begin{array}{l}\text { VR reduced all Graphic Rating } \\
\text { Scale pain scores (worst pain, time } \\
\text { spent thinking about the pain, and } \\
\text { pain unpleasantness by } 27,37 \text {, and } \\
31 \% \text {, respectively), relative to the } \\
\text { no VR condition }\end{array}$ \\
\hline $\begin{array}{l}\text { Konstantatos } \\
\text { et al, } 2009\end{array}$ & $\begin{array}{l}\text { Entertainment/ } \\
\text { Distraction } \\
\text { system }\end{array}$ & $1-$ & $\begin{array}{l}\text { Changes in patient- } \\
\text { reported pain levels }\end{array}$ & $\begin{array}{l}10 \mathrm{~cm} \text { VisualAnalogue Rating } \\
\text { Scale }\end{array}$ & $\begin{array}{l}\text { The group receiving VR relaxation } \\
\text { plus morphine PCA reported higher } \\
\text { pain intensities during the dressing } \\
\text { change (mean }=7.3) \text { compared } \\
\text { with patients receiving morphine } \\
\text { PCA alone (mean }=5.3) \\
(p=0.003)(95 \% \mathrm{Cl}: 0.6-2.8)\end{array}$ \\
\hline \multirow[t]{2}{*}{$\begin{array}{l}\text { Sharar et al, } \\
2007\end{array}$} & \multirow[t]{2}{*}{$\begin{array}{l}\text { Entertainment/ } \\
\text { Distraction } \\
\text { system }\end{array}$} & $1-$ & Patient engagement & $\begin{array}{l}\text { Sense of "presence" } \\
(0-100 \text { Graphical Analog Scale) }\end{array}$ & $\begin{array}{l}\text { Children reported higher } \\
\text { subjective reports of "presence" } \\
\text { using VR and "realness" of VR than } \\
\text { adults. Age did not affect analgesic } \\
\text { effects of VR distraction }\end{array}$ \\
\hline & & $2-$ & $\begin{array}{l}\text { Changes in patient- } \\
\text { reported pain levels }\end{array}$ & $\begin{array}{l}\text { 0-100 mm Graphical Rating } \\
\text { Scale }\end{array}$ & $\begin{array}{l}\text { Compared with standard analgesic } \\
\text { treatment alone, addition of VR } \\
\text { resulted in pain ratings reduction } \\
\text { for worst pain intensity ( } 20 \% \\
\text { reduction), pain unpleasantness } \\
\text { ( } 26 \% \text { reduction), and time spent } \\
\text { thinking about pain } \\
\text { ( } 37 \% \text { reduction) }\end{array}$ \\
\hline
\end{tabular}

(Continued) 
Table 2 (Continued)

\begin{tabular}{|c|c|c|c|c|c|}
\hline \multirow{4}{*}{$\begin{array}{l}\text { Author } \\
\text { Das et al, } \\
2005\end{array}$} & \multirow{4}{*}{$\begin{array}{l}\text { Category } \\
\text { Entertainment/ } \\
\text { Distraction } \\
\text { system }\end{array}$} & \multicolumn{4}{|c|}{ Outcome measures } \\
\hline & & \multicolumn{2}{|c|}{ Main outcome } & \multirow{2}{*}{$\begin{array}{l}\text { Measurement } \\
\text { Interviews with nurses, parents } \\
\text { and children }\end{array}$} & \multirow{2}{*}{$\begin{array}{l}\text { Main findings } \\
\text { General positive feedback regard- } \\
\text { ing the effects of VR in distraction, } \\
\text { which had an influence in reducing } \\
\text { sensitivity to pain }\end{array}$} \\
\hline & & $1-$ & User satisfaction & & \\
\hline & & $2-$ & $\begin{array}{l}\text { Changes in patient- } \\
\text { reported pain levels }\end{array}$ & Modified Faces Pain Scale & $\begin{array}{l}\text { Average pain scores for pharma- } \\
\text { cological analgesia only was } 4 \text {, } \\
\text { compared with VR with pharma- } \\
\text { cological analgesia (average pain } \\
\text { score was } 1.3 \text { ) }\end{array}$ \\
\hline \multirow[t]{2}{*}{$\begin{array}{l}\text { Hoffman } \\
\text { et al, } 2000\end{array}$} & \multirow[t]{2}{*}{$\begin{array}{l}\text { Entertainment/ } \\
\text { Distraction } \\
\text { system }\end{array}$} & $1-$ & Patient engagement & $\begin{array}{l}\text { Sense of "presence" } \\
\text { (0-100 Visual Analog Scale) }\end{array}$ & $\begin{array}{l}\text { Patients reported mean presence } \\
\text { in VR was } 63.67 \mathrm{~mm} \text {, and mean } \\
\text { realism of virtual objects was } \\
51.92 \mathrm{~mm}\end{array}$ \\
\hline & & $2-$ & $\begin{array}{l}\text { Changes in patient- } \\
\text { reported pain levels }\end{array}$ & 0-100 mm Visual Analog Scale & $\begin{array}{l}\text { Patients completed } 5 \text { subjective } \\
\text { pain ratings. They reported less } \\
\text { pain, when distracted with VR, } \\
\text { magnitude of pain reduction by VR } \\
\text { was statistically significant }\end{array}$ \\
\hline \multirow[t]{2}{*}{$\begin{array}{l}\text { Hoffman } \\
\text { et al, } 2001\end{array}$} & \multirow[t]{2}{*}{$\begin{array}{l}\text { Entertainment/ } \\
\text { Distraction } \\
\text { system }\end{array}$} & $1-$ & Patient engagement & $\begin{array}{l}\text { Sense of "presence" } \\
\text { (0-100 Visual Analog Scale) }\end{array}$ & $\begin{array}{l}\text { Patients experiencing VR were } \\
\text { asked after each physical therapy } \\
\text { session. All, except one patient, } \\
\text { reported a score above } 50\end{array}$ \\
\hline & & $2-$ & $\begin{array}{l}\text { Changes in patient- } \\
\text { reported pain levels }\end{array}$ & 0-100 mm Visual Analog Scale & $\begin{array}{l}\text { Patients completed } 5 \text { subjective } \\
\text { pain ratings. Pain ratings were } \\
\text { statistically lower with VR. } \\
\text { Repeated use of VR resulted in } \\
\text { magnitude of pain reduction }\end{array}$ \\
\hline $\begin{array}{l}\text { Martorella } \\
\text { et al, } 2012\end{array}$ & $\begin{array}{l}\text { Communication/ } \\
\text { Educational } \\
\text { system }\end{array}$ & $1-$ & $\begin{array}{l}\text { Changes in patient- } \\
\text { reported pain levels }\end{array}$ & $\begin{array}{l}\text { Barriers Questionnaire-II, } \\
\text { Pain Catastrophizing Scale }\end{array}$ & $\begin{array}{l}\text { Experimental group reported less } \\
\text { pain interference when breathing/ } \\
\text { coughing }(p=0.04) \text {. Day } 7 \text { post- } \\
\text { surgery, experimental group } \\
\text { reported fewer pain-related bar- } \\
\text { riers: (mean: } 10.6) \text { vs. controls } \\
\text { (mean } 15.8)(p=0.02) \text {. } \\
\text { Both groups mean revealed lower } \\
\text { tendency to catastrophize pain } \\
\text { presurgery }\end{array}$ \\
\hline $\begin{array}{l}\text { Martorella } \\
\text { et al, } 2013\end{array}$ & $\begin{array}{l}\text { Communication/ } \\
\text { Educational } \\
\text { system }\end{array}$ & $1-$ & User satisfaction & $\begin{array}{l}\text { Questionnaires of acceptability } \\
\text { with } 10 \text { multiple-choice } \\
\text { questions }\end{array}$ & $\begin{array}{l}\text { Most participants indicated that the } \\
\text { strategies proposed responded to } \\
\text { their needs (96\%) and that informa- } \\
\text { tion provided helped control pain } \\
\text { and lessen worries }(93 \%)\end{array}$ \\
\hline \multirow[t]{3}{*}{$\begin{array}{l}\text { McDonall } \\
\text { et al, } 2019\end{array}$} & \multirow[t]{3}{*}{$\begin{array}{l}\text { Communication/ } \\
\text { Educational } \\
\text { system }\end{array}$} & $1-$ & $\begin{array}{l}\text { Changes in clinical } \\
\text { workflow }\end{array}$ & Length of hospital stay (LOS) & $\begin{array}{l}\text { Significant reduction in LOS for the } \\
\text { intervention group } 5.29 \mathrm{~d} \text { vs. } \\
\text { control group } 6.29 \mathrm{~d}(p=0.04)\end{array}$ \\
\hline & & $2-$ & Patient engagement & Patient activation measure & $\begin{array}{l}\text { No significant positive difference } \\
\text { was reported between both groups. } \\
\text { On day } 3 \text { after surgery, there was a } \\
\text { reduction in the } \\
\text { proportion of patients at level } 3 \text { and } \\
4 \text { activation in both groups from } \\
\text { baseline scores (prior to } \\
\text { admission). Control group } \\
\text { significantly higher ( } 20 \% \text { decrease) }\end{array}$ \\
\hline & & $3-$ & User satisfaction & $\begin{array}{l}\text { Global satisfaction questions } \\
\text { Net Promoter Score (recommend } \\
\text { the health service to a family or } \\
\text { friend) }\end{array}$ & $\begin{array}{l}\text { Intervention group reported } \\
\text { significantly higher mean satisfac- } \\
\text { tion with acute care experience } \\
9.26 \text { versus } 8.58 \text { control group } \\
(p=0.01) \text { and higher NET } \\
\text { promoter score } 9.27 \text { versus } \\
8.67 \text { control group }(p=0.02)\end{array}$ \\
\hline
\end{tabular}




\begin{tabular}{|c|c|c|c|c|c|}
\hline \multirow[t]{3}{*}{ Author } & \multirow[t]{3}{*}{ Category } & \multicolumn{4}{|c|}{ Outcome measures } \\
\hline & & \multicolumn{2}{|c|}{ Main outcome } & \multirow[b]{2}{*}{$\begin{array}{l}\text { Measurement } \\
\text { Pain intensity (Numerical Rating } \\
\text { Scale 0-10), } \\
\text { Pain quality (American Pain } \\
\text { Society Outcome Questionnaire- } \\
\text { Revised version), and Pain } \\
\text { treatment- } \\
\text { analgesic management } \\
\text { (medical record audit) }\end{array}$} & \multirow[b]{2}{*}{$\begin{array}{l}\text { Main findings } \\
\text { Postoperative day } 3: \\
\text { Pain intensity: significant reduc- } \\
\text { tion }(1.01) \text { in mean pain scores } \\
\text { between groups. } 64 \% \text { of controls } \\
\text { had a day } 3 \text { score } \geq 7, \text { compared } \\
\text { with } 44 \% \text { of intervention group } \\
(p=0.04) \text {. } \\
\text { Pain quality: interventions } \\
\text { perceived they received more pain } \\
\text { relief in previous } 24 \mathrm{~h} \\
\text { (mean }=7.67 \text { vs. mean }=7.07 \\
(p=0.05) . \text { Significantly more } \\
\text { intervention patients reported } \\
\text { using deep breathing to relieve } \\
\text { pain ( } 82.2 \% \text { vs. } 68 \%)(p=0.02) . \\
\text { Pain treatment: intervention } \\
\text { group received significantly higher } \\
\text { amounts of pro re } \\
\text { nata oxycodone (daily amount } \\
\text { (mg) - mean }=16.1 \text { vs. control } \\
\text { group } 10.8, p=0.001)\end{array}$} \\
\hline & & 4- & $\begin{array}{l}\text { Changes in patient- } \\
\text { reported pain levels }\end{array}$ & & \\
\hline \multirow[t]{4}{*}{$\begin{array}{l}\text { Wirz et al, } \\
2017\end{array}$} & \multirow[t]{4}{*}{$\begin{array}{l}\text { Personalized } \\
\text { PCA device }\end{array}$} & $1-$ & $\begin{array}{l}\text { Changes in clinical } \\
\text { workflow }\end{array}$ & $\begin{array}{l}\text { Value-stream mapping } \\
\text { (comparison between pre- and } \\
\text { postimplementation) }\end{array}$ & $\begin{array}{l}\text { Medication provision process preim- } \\
\text { plementation comprised of } 8 \text { steps. } \\
\text { Postimplementation was } 3 \text { steps }\end{array}$ \\
\hline & & $2-$ & Patient engagement & $\begin{array}{l}\text { "Efficacy measure"-data } \\
\text { recorded by the device for each } \\
\text { patient and questionnaires filled } \\
\text { out by patients }\end{array}$ & $\begin{array}{l}\text { Success rate of } 90 \% \text { for pill intake } \\
\text { upon patient's request }\end{array}$ \\
\hline & & 3- & User satisfaction & $\begin{array}{l}\text { "Usability measure" measured } \\
\text { using questionnaires filled out by } \\
\text { patients and medical staff }\end{array}$ & $\begin{array}{l}\text { At least } 80 \% \text { of patients and } \\
\text { medical staff were satisfiedwith } \\
\text { device use and recommend its use }\end{array}$ \\
\hline & & 4- & $\begin{array}{l}\text { Changes in patient- } \\
\text { reported pain levels }\end{array}$ & 0-10 Numeric Rating Scale & $\begin{array}{l}\text { Patients reported pain levels } \\
\text { before and after pill intake using } \\
\text { PCA. They reported significantly } \\
\text { less pain, both at rest and in } \\
\text { movement, from first } \\
\text { postoperative day }\end{array}$ \\
\hline \multirow[t]{3}{*}{$\begin{array}{l}\text { Aldekhyyel } \\
\text { et al, } 2018\end{array}$} & \multirow[t]{3}{*}{$\begin{array}{l}\text { Interactive } \\
\text { Platform } \\
\text { System }\end{array}$} & $1-$ & Changes in documentation & $\begin{array}{l}\% \text { pain documentation } \\
\text { occurrences (comparison } \\
\text { between pre- and } \\
\text { postimplementation) }\end{array}$ & $\begin{array}{l}\text { Modest increase in mean timely } \\
\text { documentation rates based on } \\
\text { nursing documentation standards } \\
\text { ( } 26.1 \% \text { vs. } 32.8 \% \text {, a } \% \text { increase of } \\
25.7 \% ; p<0.001 \text { ) along with } \\
\text { decreased median time to pain } \\
\text { reassessment documentation } \\
\text { ( } 29 \text { min vs. } 25 \text { min, \% decrease of } \\
13.8 \% ; p<0.001)\end{array}$ \\
\hline & & $2-$ & $\begin{array}{l}\text { Changes in clinical } \\
\text { workflow }\end{array}$ & $\begin{array}{l}\text { Value-stream mapping } \\
\text { (comparison between pre- and } \\
\text { postimplementation) }\end{array}$ & $\begin{array}{l}\text { Pain management tool interfaced } 4 \\
\text { standalone technologies. The work- } \\
\text { flow is triggered when pain medi- } \\
\text { cations are dispensed by sending an } \\
\text { automatic pain assessment rating } \\
\text { question via the patient's television } \\
\text { at a predefined time }\end{array}$ \\
\hline & & $3-$ & Patient engagement & $\begin{array}{l}\text { Usage rates based on responses } \\
\text { to system prompts }\end{array}$ & $\begin{array}{l}\text { Usage rates were low with } 6.5 \% \text { for } \\
\text { the level of pain prompt and } 13.3 \% \\
\text { for the other nonpharmacologic } \\
\text { strategies to help with pain prompt }\end{array}$ \\
\hline $\begin{array}{l}\text { Aldekhyyel } \\
\text { et al, } 2018\end{array}$ & $\begin{array}{l}\text { Interactive } \\
\text { Platform } \\
\text { System }\end{array}$ & \multicolumn{2}{|c|}{ 1- User satisfaction } & $\begin{array}{l}2 \text { survey instruments: closed- } \\
\text { ended ( } 5 \text { point Likert scale) and } \\
\text { open-ended questions }\end{array}$ & $\begin{array}{l}\text { Parents were more satisfied (90\%) } \\
\text { compared with nurses ( } 50 \%) \text {. } \\
\text { Timely reassessments of pain were } \\
\text { the most valuable feature } \\
\text { reported. Qualitative analysis of } \\
\text { nurses' responses yielded } 6 \\
\text { themes for system benefits and } 12 \\
\text { for challenges }\end{array}$ \\
\hline
\end{tabular}


Table 2 (Continued)

\begin{tabular}{|c|c|c|c|c|c|}
\hline \multirow{3}{*}{$\begin{array}{l}\text { Author } \\
\text { Patmon } \\
\text { et al, } 2016\end{array}$} & \multirow{3}{*}{$\begin{array}{l}\text { Category } \\
\text { Interactive } \\
\text { Platform } \\
\text { System }\end{array}$} & \multicolumn{4}{|c|}{ Outcome measures } \\
\hline & & \multicolumn{2}{|c|}{ Main outcome } & \multirow{2}{*}{$\begin{array}{l}\text { Measurement } \\
\text { Interviews using a focused rapid } \\
\text { ethnographic evaluation }\end{array}$} & \multirow[b]{2}{*}{$\begin{array}{l}\text { Main findings } \\
\text { Participants reported findings of } \\
\text { using the system effective for } \\
\text { distraction, great functionality for } \\
\text { patients and nurses, has implica- } \\
\text { tions for clinical practice, and } \\
\text { needs additional training to } \\
\text { improve usage }\end{array}$} \\
\hline & & $1-$ & User satisfaction & & \\
\hline \multirow[t]{3}{*}{$\begin{array}{l}\text { Rao-Gupta } \\
\text { et al, } 2018\end{array}$} & \multirow[t]{3}{*}{$\begin{array}{l}\text { Interactive } \\
\text { Platform } \\
\text { System }\end{array}$} & $1-$ & $\begin{array}{l}\text { Changes in clinical } \\
\text { workflow }\end{array}$ & $\begin{array}{l}\text { Value-stream mapping } \\
\text { (comparison between pre- and } \\
\text { postimplementation) }\end{array}$ & $\begin{array}{l}\text { New workflow included } 2 \text { main } \\
\text { improvements: admission assess- } \\
\text { ment questions for healthcare } \\
\text { team and integrations between } \\
\text { interactive tool with EHR and } \\
\text { medication dispensing system }\end{array}$ \\
\hline & & $2-$ & User satisfaction & $\begin{array}{l}\text { Proportion of "always" responses } \\
\text { to the (Child HCAHPS, } \\
\text { 2014-2016) pain question }\end{array}$ & $\begin{array}{l}\text { Proportion of family satisfaction } \\
\text { responses to the question "Did } \\
\text { staff do everything they could to } \\
\text { manage your child's pain?" } \\
\text { increased from year } 2014 \text { to } \\
\text { year } 2016(p=0.006)\end{array}$ \\
\hline & & $3-$ & Changes in documentation & \# documentation occurrences & $\begin{array}{l}\text { Documentation of nonpharmaco- } \\
\text { logic interventions: year } 2014 \\
\text { (\# unique patients }=2,462 \text { ), } \\
\text { year } 2015 \text { (\# unique patients } \\
=2,684 \text { ), year } 2016 \\
\text { (\# unique patients }=2,970 \text { ) }\end{array}$ \\
\hline
\end{tabular}

Abbreviations: $\mathrm{Cl}$, confidence interval; EHR, electronic health record; GI, gastrointestinal; PCA, patient-controlled analgesia; VR, virtual reality.

study that reported that use of VR-guided relaxation during dressings changes resulted in higher pain scores when compared with the use of morphine alone $(p=0.003)(95 \%$ confidence interval: $0.6-2.8){ }^{40}$

In addition to reporting changes in patient-reported pain levels as a main outcome measure, four VR intervention studies reported levels of patient engagement and one reported levels of patient satisfaction as a secondary outcome measure. Studies that reported levels of patient engagement $^{30,36-38}$ used a 1 to 100 Visual or Graphical Analog scale to measure sense of presence "illusion of being inside the computer-generated environment" 30 and engagement while using the VR system. ${ }^{30}$ Hoffman et al reported average engagement mean scores in both of their studies. $^{30,38}$ Carrougher et al showed that approximately half of the adult burn patients rated their level of presence and engagement with the VR during physical therapy treatment, at lower average scores, ${ }^{36}$ while Sharar et al showed that children reported higher levels of engagement than adults. ${ }^{37}$

Among this group, only one study reported levels of satisfaction with the use of VR as a secondary outcome. The authors measured levels of satisfaction through a series of interviews with parents and nurses. Nursing staff indicated that the use of VR during wound dressing changes was helpful in distracting children from pain and "all parents agreed with the positive effects of VR in pain management for their child. They all commented that the child's anxiety level was perceptibly less when using VR, and the child looked forward to playing the VR game." 41

The interactive music intervention study measured pain scores at baseline and three posttests reporting on the Pain
Rating Index (PRI-total) score from baseline. Music therapy was found to reduce the PRI-total score in the intervention group compared with the control group. ${ }^{42}$

\section{Communication/Educational Systems}

The two studies published by Martorella et al describing the use of a virtual nursing Web-based intervention for selfmanagement of pain postcardiac surgery, reported changes in patient pain levels using the Barriers Questionnaire-II. ${ }^{43}$ Patients in the intervention group reported fewer pain-related barriers (mean: 10.6) than patients in the control group (mean: 15.8, $p=0.02$ ). In their follow-up study, the authors reported the acceptability of the virtual nursing Web-based intervention, by measuring the perceptions of patients using a questionnaire. ${ }^{51}$ Most of the patients indicated that the strategies proposed responded to their needs and that the information provided helped control pain and lessen worries.

A recent study by McDonall et al evaluated the efficacy of a clinician-facilitated, bedside multimedia intervention for patients recovering from total knee replacement surgery. ${ }^{44}$ The study described changes in pain-reported outcomes postoperative day 3 as a main outcome measure using three different measures: pain intensity (Numerical Rating Scale), pain quality (American Pain Society Outcome QuestionnaireRevised), and pain treatment analgesic management (medical record audit). Patients in the intervention group reported a lower mean worst pain score (6.05) than the control group $(7.05, p=0.04)$. Secondary outcomes included patient activation, length of hospital stay, knee function, and satisfaction with care. The intervention group had lower length of stay (5.29 days vs. 6.29 days, $p=0.04$ ), higher levels of patient 
activation (45.1\% level 4 activation vs. $27.1 \%, p=0.04$ ), and higher mean satisfaction with acute care experience (9.26 vs. 8.58, $p=0.01$ ).

\section{Personalized PCA Device}

The study of the oral PCA dispenser reported an improvement in the clinical workflow from a total of eight to three steps. The authors also reported three secondary outcome measures: (1) high level of patient engagement (success rate for using the device of 90\%); (2) lower patient-reported pain levels during day 1 and day 2 postoperative during rest (33.56\% reduction, $p=0.0058$ ) and movement ( $28 \%$ reduction, $p=0.0012$ ); and (3) high satisfaction with the usability of the device as indicated by both patients and medical staff ( $80 \%$ were satisfied). ${ }^{45}$

\section{Interactive Platform Systems}

Two studies describing the implementation an interactive pain management platform system accessed through inpatient television screens, reported improvements in patient pain reassessment nursing documentation practices by calculating the percent of pain documentation occurrences pre- and postsystem implementation. The authors also reported: (1) improvement in clinical workflow by implementing nursing automatic alerts to conduct timely reassessments of pain, and (2) low levels of patient engagement with the system by calculating system usage rates. ${ }^{47}$ In their follow-up study, the authors captured the perceptions of nurses and parents with the use of the system. Parents were more satisfied with the experience compared with nurses and both nurses and parents indicated that timely reassessments of pain was the most valuable system feature. ${ }^{48}$

A second group of authors described a quality improvement project to integrate a similar pain management interactive system with the hospital's EHR and medication dispensing system. The authors reported an increase in patients' satisfaction scores with the hospital's pain management initiatives, as a result of the integration. The authors also reported an increase in documentation of nonpharmacologic interventions. ${ }^{49}$

Patmon et al interviewed nurses to capture their perceptions with using an interactive patient engagement technology during their daily clinical practice. Nurses reported effectiveness of the tool for distraction therapy, satisfaction with the functionality of the tool, positive implications for clinical practice, and the need for additional training to improve usage. ${ }^{50}$

\section{Discussion}

Our systematic review identified the types of patient interactive systems used in the inpatient setting for the management of pain and the impact of these systems on controlling patient pain levels, hospital workflows, patient engagement, and user satisfaction. The majority of the studies included in our review were VR systems used for distracting burn patients during physical therapy treatment or wound dressing changes. These types of studies mainly reported the effects of using VR systems on controlling patient pain levels during hospitalization. These systems along with the communication/educational systems, which were included in our review, have shown to be effective in managing pain levels for patients.

With recently published studies, we found that there is a smaller but growing body of evidence describing the use of interactive platform systems promoting self-management of pain, increasingly with integration into different hospital technology systems. These interactive systems earned positive feedback from users, increased levels of satisfaction, and resulted in improved clinical workflows. Our review also revealed that interactive platform systems designed to support more than one aspect of pain management during hospitalization can potentially align with national pain management standards. Specifically, these systems may assist with increasing patient engagement in pain management treatment planning through education, providing nonpharmacologic pain treatment modalities, and facilitating reassessment and timely responses to patient's pain through automatic documentation of response(s) to pain interventions. ${ }^{2}$ Additionally, some studies demonstrated the feasibility of integrating these systems with the EHR to support clinical documentation, create standardized fields for patient-generated pain assessment data, and allow patients to access their records. Having the ability to integrate these systems with the EHR system, taking into account a standardized pain information model, ${ }^{52,53}$ may potentially be of great value for health care organizations seeking the adoption of these interactive pain management systems, support secondary use for research purposes, ${ }^{54}$ and create an opportunity to study the effects of these systems on reducing patient pain levels.

While the different interactive pain management platform systems included in our review have been well received by users, the number of patients/parents and nurses that used these systems was relatively low. To determine areas of improvement and enhance the systems' use among both patients/parents and clinicians/nurses, it is essential to conduct a usability evaluation study. ${ }^{55-57}$ Expanding the use of these interactive platform systems for the management of other conditions may have the potential to support many patient engagement initiatives. ${ }^{58-62}$

Limitations of this review include the different definitions of interactivity, patient engagement, and outcome measures, making direct comparisons difficult. The differences in definitions, populations, and study designs led to heterogeneity, which in turn made a meta-analysis infeasible. This lack of a meta-analysis limits our ability to draw conclusions regarding the efficiency, efficacy, and effectiveness of one type of pain interactive system when compared with a different type of system. This is especially true when examining entertainment/distraction systems versus interactive platform systems, due to lack of research studies that report the effects of interactive platform systems in managing patient pain levels.

Another limitation is related to the sample sizes of some of our reviewed studies. Four of the nine reviewed VR studies had a very small sample size ranging from 7 to 12 patients. The 
reduced sample size limits the ability to generalize the results of these studies, and further research with a larger sample size may provide better understanding of the usefulness and effectiveness of those specific VR systems as a nonpharmacological approach to pain relief.

The impossibility of blinding participants and assessors in these studies also introduces the possibility of bias. Some studies lacked the specific details related to addressing the risk of bias, while other studies entirely did not mention it. This in turn resulted in an unclear risk of bias in the majority of the studies included in our review.

Finally, the five outcome measure themes described in our review were not equally represented in the literature and were heterogeneous within outcome measure themes. Limiting our review to those articles describing only pain management interactive patient care systems and its application in the inpatient care setting likely influenced the representation of outcome measures we observed. By restricting our review, we may have also unintentionally removed meaningful details from other patient interactive systems.

We also observed a diversity in evaluation criteria and a lack of a standardized framework for measuring the efficiency and effectiveness of interactive pain management interactive systems. Improved standardized evaluation assessments will help to improve our ability broadly to assess and improve health outcomes in existing and future pain management interactive systems.

\section{Conclusion}

The use of inpatient interactive systems in the management of pain is an emerging area of interest for researchers and health care providers in the era of modern health care technology and increased focus on patient engagement. These systems have primarily been entertainment-focused and built to distract patients during a treatment procedure and have shown an impact in lowering levels of patientreported pain scores. Inpatient interactive pain management systems integrated with the hospital's EHR further facilitate patient-provider communication, patient education, and self-reporting; they show promising effects on timely pain assessments, increasing patient satisfaction, and patient engagement. Further high-quality studies with a more standardized approach to implementation and assessment are necessary to reinforce and validate these findings.

\section{Clinical Relevance Statement}

Addressing a hospitalized patient's pain through the use of other nonpharmacological measures has been the focus of many national standards and is an essential part of clinical care. Patient interactive systems continue to evolve in support of a more patient-centric approach to the management of pain. Findings of this study contribute to research efforts that evaluate the use of health information technology systems and their impact on patient care.

\section{Multiple Choice Questions}

1. Which of the following can be considered one of the benefits of integrating patient interactive systems with the hospital's EHR system?

a. Increasing security and privacy measures.

b. Enhancing the quality of data being stored.

c. Collecting and reporting quality indicators.

d. Maintaining active patients list.

Correct Answer: The correct answer is option b. Inpatient interactive systems can be designed to automatically collect and store patient-generated reported outcomes. Having these systems integrated with the EHR has the potential to meet regulatory compliance standards and ultimately enhance the quality of data being stored in the EHRs for secondary quality use.

2. Which of the following is defined as the "extent to which users can participate in modifying the form and content of a mediated environment in real time"?

a. Interoperability.

b. Integration.

c. Interactivity.

d. Incorporation.

Correct Answer: The correct answer is option C. Steuer was considered one of the early scholars that defined interactivity by focusing on the process of real-time participation between the mediated environment and the users.

Protection of Human and Animal Subjects

Not applicable.

Funding

This research was supported by the National Institutes of Health National Center for Advancing Translational Sciences (UL1TR000114).

\section{Conflict of Interest}

None declared.

\section{References}

1 Hospital HCAHPS. Published 2017. Available at: https://www.cms. gov/Medicare/Quality-Initiatives-Patient-Assessment-instruments/ hospitalqualityinits/hospitalHCAHPS.html. Accessed February 1, 2019

2 The Joint Commission. R3 Report | Requirement, Rationale, Reference; 2017. Available at: www.jointcommission.org. Accessed February 1, 2019

3 Wandner LD, Scipio CD, Hirsh AT, Torres CA, Robinson ME. The perception of pain in others: how gender, race, and age influence pain expectations. J Pain 2012;13(03):220-227

4 Anderson KO, Mendoza TR, Valero V, et al. Minority cancer patients and their providers: pain management attitudes and practice. Cancer 2000;88(08):1929-1938

5 Rhudy JL, Meagher MW. Fear and anxiety: divergent effects on human pain thresholds. Pain 2000;84(01):65-75

6 Linton SJ, Shaw WS. Impact of psychological factors in the experience of pain. Phys Ther 2011;91(05):700-711 
7 Hanna MN, González-Fernández M, Barrett AD, Williams KA, Pronovost $P$. Does patient perception of pain control affect patient satisfaction across surgical units in a tertiary teaching hospital? Am J Med Qual 2012;27(05):411-416

8 de Rond ME, de Wit R, van Dam FS, Muller MJ. A pain monitoring program for nurses: effects on communication, assessment and documentation of patients' pain. J Pain Symptom Manage 2000;20 (06):424-439

9 Samuels JG, Kritter D. Pain management documentation. Comput Informatics Nurs 2011;29(09):512-518

10 Sikka K, Ahmed AA, Diaz D, et al. Automated assessment of children's postoperative pain using computer vision. Pediatrics 2015;136(01):e124-e131

11 Symer MM, Abelson JS, Milsom J, McClure B, Yeo HL. A mobile health application to track patients after gastrointestinal surgery: results from a pilot study. J Gastrointest Surg 2017;21(09): 1500-1505

12 Agboola S, Kamdar M, Flanagan C, et al. Pain management in cancer patients using a mobile app: study design of a randomized controlled trial. JMIR Res Protoc 2014;3(04):e76

13 Neary PM, Sung R, Corrigan M, O'Donovan M, Cahill RA, Redmond HP. The benefits of an interactive, individualized online patient pathway for patients undergoing minimally invasive radioguided parathyroidectomy: a prospective, double-blinded, randomized clinical trial. Surg Innov 2010;17(03):236-241

14 Riva S, Camerini A-L, Allam A, Schulz PJ. Interactive sections of an Internet-based intervention increase empowerment of chronic back pain patients: randomized controlled trial. J Med Internet Res 2014;16(08):e180

15 Steuer J. Defining virtual reality: dimensions determining telepresence. J Commun 1992;42(04):73-93

16 Hibbard JH, Greene J. What the evidence shows about patient activation: better health outcomes and care experiences; fewer data on costs. Health Aff (Millwood) 2013;32(02):207-214

17 Fisher B, Britten N. Patient access to records: expectations of hospital doctors and experiences of cancer patients. Br J Gen Pract 1993;43(367):52-56

18 Maly RC, Bourque LB, Engelhardt RF. A randomized controlled trial of facilitating information giving to patients with chronic medical conditions: effects on outcomes of care. J Fam Pract 1999;48(05): 356-363

19 Prey JE, Woollen J, Wilcox L, et al. Patient engagement in the inpatient setting: a systematic review. J Am Med Inform Assoc 2014;21(04):742-750

20 Tang C, Lorenzi N, Harle CA, Zhou X, Chen Y. Interactive systems for patient-centered care to enhance patient engagement. J Am Med Inform Assoc 2016;23(01):2-4

21 Pal K, Eastwood SV, Michie S, et al. Computer-based diabetes selfmanagement interventions for adults with type 2 diabetes mellitus. Cochrane Database Syst Rev 2013;(03):CD008776

22 Marcano Belisario JS, Huckvale K, Greenfield G, Car J, Gunn LH. Smartphone and tablet self management apps for asthma. Cochrane Database Syst Rev 2013;(11):CD010013

23 Wieland LS, Falzon L, Sciamanna CN, et al. Interactive computerbased interventions for weight loss or weight maintenance in overweight or obese people. Cochrane Database Syst Rev 2012; (08):CD007675

24 Rigotti NA, Clair C, Munafò MR, Stead LF. Interventions for smoking cessation in hospitalised patients. Cochrane Database Syst Rev 2012;(05):CD001837

25 Raniah Aldekhyyel, Michael Pitt, Caitlin Bakker, Genevieve Melton. The impact of interactive patient care tools used for the management of pain in an inpatient setting: a systematic review. PROSPERO 2018 CRD42018100105 Available at: http://www.crd. york.ac.uk/PROSPERO/display_record.php?ID=CRD42018100105. Accessed July 24, 2019

26 Higgins JP, Lasserson T, Chandler J, Tovey D, Churchill R. Methodological Expectations of Cochrane Intervention Reviews (MECIR)
Standards for the Conduct and Reporting of New Cochrane Intervention Reviews, Reporting of Protocols and the Planning, Conduct and Reporting of Updates; 2016. Available at: https://community. cochrane.org/sites/default/files/uploads/MECIR PRINTED BOOKLET FINAL v1.01.pdf. Accessed February 5, 2019

27 Clarivate Analytics, EndNote; 2016. Available at: https://clarivate. $\mathrm{com} /$ product-category/scientific-academic-research/. Accessed July 21, 2019

28 Ouzzani M, Hammady H, Fedorowicz Z, Elmagarmid A. Rayyan-a web and mobile app for systematic reviews. Systematic Reviews 2016;5:210. Doi: 10.1186/s13643-016-0384-4

29 Moher D, Liberati A, Tetzlaff J, Altman DG; PRISMA Group. Preferred reporting items for systematic reviews and meta-analyses: the PRISMA statement. PLoS Med 2009;6(07):e1000097

30 Hoffman HG, Patterson DR, Carrougher GJ. Use of virtual reality for adjunctive treatment of adult burn pain during physical therapy: a controlled study. Clin J Pain 2000;16(03): 244-250

31 Sterne JA, Hernán MA, Reeves BC, et al. ROBINS-I: a tool for assessing risk of bias in non-randomised studies of interventions. BMJ 2016;355(October):i4919

32 Higgins JPT, Altman DG, Gøtzsche PC, et al; Cochrane Bias Methods Group; Cochrane Statistical Methods Group. The Cochrane Collaboration's tool for assessing risk of bias in randomised trials. BMJ 2011;343(02):d5928-d5928

33 Qualtrics. 2018. Avaliable at: https://www.qualtrics.com. Accessed July 21, 2019

34 Morris LD, Louw QA, Crous LC. Feasibility and potential effect of a low-cost virtual reality system on reducing pain and anxiety in adult burn injury patients during physiotherapy in a developing country. Burns 2010;36(05):659-664

35 Tashjian VC, Mosadeghi S, Reid MW, Howard A, Lopez M, Spiegel B. Virtual reality reduces abdominal pain in hospitalized patients: results of a controlled trial. Gastroenterology 2017. Doi: 10.1016/ S0016-5085(17)30727-8

36 Carrougher GJ, Hoffman HG, Nakamura D, et al. The effect of virtual reality on pain and range of motion in adults with burn injuries. J Burn Care Res 2009;30(05):785-791

37 Sharar SR, Carrougher GJ, Nakamura D, Hoffman HG, Blough DK, Patterson DR. Factors influencing the efficacy of virtual reality distraction analgesia during postburn physical therapy: preliminary results from 3 ongoing studies. Arch Phys Med Rehabil 2007; 88(12, Suppl 2):S43-S49

38 Hoffman HG, Patterson DR, Carrougher GJ, Sharar SR. Effectiveness of virtual reality-based pain control with multiple treatments. Clin J Pain 2001;17(03):229-235

39 Faber AW, Patterson DR, Bremer M. Repeated use of immersive virtual reality therapy to control pain during wound dressing changes in pediatric and adult burn patients. J Burn Care Res 2013;34(05):563-568

40 Konstantatos AH, Angliss M, Costello V, Cleland H, Stafrace S. Predicting the effectiveness of virtual reality relaxation on pain and anxiety when added to PCA morphine in patients having burns dressings changes. Burns 2009;35(04):491-499

41 Das DA, Grimmer KA, Sparnon AL, McRae SE, Thomas BH. The efficacy of playing a virtual reality game in modulating pain for children with acute burn injuries: a randomized controlled trial [ISRCTN87413556]. BMC Pediatr 2005;5(01):1

42 Li XM, Yan H, Zhou KN, Dang SN, Wang DL, Zhang YP. Effects of music therapy on pain among female breast cancer patients after radical mastectomy: results from a randomized controlled trial. Breast Cancer Res Treat 2011;128(02):411-419

43 Martorella G, Côté J, Racine M, Choinière M. Web-based nursing intervention for self-management of pain after cardiac surgery: pilot randomized controlled trial. J Med Internet Res 2012;14 (06):e177

44 McDonall J, de Steiger R, Reynolds J, et al. Patient activation intervention to facilitate participation in recovery after total 
knee replacement (MIME): a cluster randomised cross-over trial. BMJ Qual Saf 2019:pii:bmjqs-2018-008975

45 Wirz S, Conrad S, Shtrichman R, Schimo K, Hoffmann E. Clinical evaluation of a novel technology for oral patient-controlled analgesia, the $P C O A \circledast$ Acute device, for hospitalized patients with postoperative pain, in pilot feasibility study. Pain Res Manag 2017;2017:7962135

46 Interactive Patient Systems; 2017 Turning Interactivity into Outcomes - KLAS Report. Available at: https://klasresearch.com/report/ interactive-patient-systems-2017/1234. Accessed May 26, 2019

47 Aldekhyyel RN, Melton GB, Lindgren B, Wang Y, Pitt MB. Linking pediatrics patients and nurses with the pharmacy and electronic health record system through the inpatient television: a novel interactive pain-management tool. Hosp Pediatr 2018;8(09):588-592

48 Aldekhyyel RN, Melton GB, Hultman G, Pitt MB. Using a bedside interactive technology to solicit and record pediatric pain reassessments: parent and nursing perspectives on a novel workflow. AMIA Jt Summits Transl Sci Proc 2018;2017:300-309

49 Rao-Gupta S, Kruger D, Leak LD, Tieman LA, Manworren RCB. Leveraging interactive patient care technology to improve pain management engagement. Pain Manag Nurs 2018;19(03):212-221

50 Patmon FL, Gee PM, Rylee TL, Readdy NL. Using interactive patient engagement technology in clinical practice: a qualitative assessment of nurses' perceptions. J Med Internet Res 2016;18(11):e298

51 Martorella G, Côté J, Choinière M. SOULAGE-TAVIE: development and validation of a virtual nursing intervention to promote selfmanagement of postoperative pain after cardiac surgery. Comput Inform Nurs 2013;31(04):189-197

52 Westra BL, Johnson SG, Ali S, et al. Validation and refinement of a pain information model from EHR flowsheet data. Appl Clin Inform 2018;9(01):185-198
53 Gesner E, Collins SA, Rocha R. Pain documentation: validation of a reference model. Stud Health Technol Inform 2015;216:805-809

54 Westra BL, Christie B, Johnson SG, et al. Modeling flowsheet data to support secondary use. Comput Inform Nurs 2017;35(09):452-458

55 Genes N, Kim MS, Thum FL, et al. Usability evaluation of a clinical decision support system for geriatric ED pain treatment. Appl Clin Inform 2016;7(01):128-142

56 Couture B, Lilley E, Chang F, et al. Applying user-centered design methods to the development of an mHealth application for use in the hospital setting by patients and care partners. Appl Clin Inform 2018;9(02):302-312

57 Roberts S, Chaboyer W, Gonzalez R, Marshall A. Using technology to engage hospitalised patients in their care: a realist review. BMC Health Serv Res 2017;17(01):388

58 Careyva B, Shaak K, Mills G, et al. Implementation of technologybased patient engagement strategies within practice-based research networks. J Am Board Fam Med 2016;29(05):581-591

59 Carman KL, Dardess P, Maurer M, et al. Patient and family engagement: a framework for understanding the elements and developing interventions and policies. Health Aff (Millwood) 2013;32(02):223-231

60 Sharma AE, Rivadeneira NA, Barr-Walker J, Stern RJ, Johnson AK, Sarkar U. Patient engagement in health care safety: an overview of mixed-quality evidence. Health Aff (Millwood) 2018;37(11): 1813-1820

61 Bates DW, Singh H. Two decades since to err is human: an assessment of progress and emerging priorities in patient safety. Health Aff (Millwood) 2018;37(11):1736-1743

62 Schwappach DLB. Review: engaging patients as vigilant partners in safety: a systematic review. Med Care Res Rev 2010;67(02): 119-148 